\title{
Étude de réactions radicalaires à très basses températures. Implications astrochimiques
}

\author{
B.R. Rowe et A. Canosa \\ Physique des Atomes, Lasers, Molécules et Surfaces, URA 1203 du CNRS, Université \\ de Rennes I, Campus de Beaulieu, 35042 Rennes cedex, France
}

\begin{abstract}
Résumé: La cinétique de nombreuses réactions radical-molécule a été étudiée à très basse température $(13-300 \mathrm{~K})$ en associant à la technique CRESU (Cinétique de Réaction en Ecoulement Supersonique Uniforme) la méthode PLP - LIF (Pulsed Laser Photolysis - Laser Induced Fluorescence) pour produire et détecter les radicaux.
\end{abstract}

\section{INTRODUCTION}

Un des problèmes les plus passionnants de l'astrophysique moderne est certainement celui de la genèse des étoiles et de leur éventuel cortège planétaire à travers l'effondrement d'un nuage interstellaire dense [1]. Le formidable développement de la radioastronomie depuis le début des années 70 et plus récemment des observatoires spatiaux tel ISO (Infrared Space Observatory) a permis d'accumuler un très grand nombre de données sur le milieu interstellaire (MIS ci-après) et de mettre en évidence, en particulier dans les nuages denses, l'existence de molécules complexes, souvent organiques. La compréhension de l'évolution de la composition chimique observée dans les atmosphères des planètes du système solaire (et de certains de leurs satellites comme Titan ou Triton) depuis leur formation dans la nébuleuse planétaire jusqu'à nos jours constituerait bien entendu une avancée décisive.

Les modèles astrochimiques qui tentent de reproduire, par le calcul, l'évolution des espèces observées, aussi bien dans le MIS que dans les atmosphères planétaires, sont devenus de plus en plus sophistiqués, incluant des réactions en phase gazeuse d'électrons, d'ions et de neutres ainsi que les réactions hétérogènes des mêmes espèces à la surface des grains [2]. Malgré cela, nous sommes encore très loin du but et de nombreuses incertitudes demeurent, en particulier dans le domaine de la chimie interstellaire. En effet, l'étude en laboratoire des processus réactionnels aux températures extrêmement faibles du MIS $(10-100 \mathrm{~K})$ constitue un véritable défi expérimental.

En dépit de cette difficulté, les années 80 ont vu de remarquables avancées dans le domaine des réactions ion-molécule, d'une part parce qu'elles sont supposées jouer un rôle clé dans la chimie interstellaire, d'autre part parce que les ions peuvent être facilement manipulés ou stockés par des champs électromagnétiques convenables et détectés avec une très grande efficacité. Ces propriétés sont à la base des techniques de tubes à dérive [3], de pièges à ions [4,5] refroidis cryogéniquement et de faisceaux colinéaires [6] mis au point par D. Gerlich pour l'étude des sections efficaces à de très faibles énergies de collision dans le centre de masse. Les techniques utilisant le refroidissement cryogénique à l'hélium sont essentiellement restreintes à l'hydrogène 
moléculaire comme réactant neutre, les autres espèces étant susceptibles de se condenser sur les parois des appareillages.

Un autre type de technique utilise des expansions supersoniques comme réacteur chimique en écoulement. Ainsi, au début des années 80 , B. Rowe et ses collaborateurs [7] ont développé une technique connue sous l'acronyme CRESU (Cinétique de Réaction en Ecoulement Supersonique Uniforme) et utilisant les jets supersoniques uniformes issus de tuyères de Laval. De très nombreuses réactions ont été étudiées par cette méthode jusqu'à $8 \mathrm{~K}$. Plus récemment, le groupe de $\mathrm{M}$. Smith a utilisé des jets libres pulsés pour étudier les réactions ion-molécule jusqu'à la 'température' record de $0.1 \mathrm{~K}$ [8]. Il s'agit ici d'une température équivalente, les conditions dans ce type de jet étant très éloignées de l'équilibre thermodynamique, ce qui peut se révéler un sérieux inconvénient dans tous les cas où le coefficient de vitesse est fortement dépendant de la population des états internes (rotation, spin-orbite) et lorsque celle ci ne peut être précisée.

Toutes ces techniques ont permis l'étude d'un grand nombre de réactions ionmolécule. En particulier, la technique CRESU a été largement utilisée pour fournir un ensemble de données sur les réactions d'ions avec des molécules fortement dipolaires, en général extrêmement condensables dans le domaine des températures explorées. A partir de l'ensemble de ces résultats expérimentaux, il a été possible de montrer que les valeurs des coefficients de vitesse à température ultra basse, pour l'essentiel des réactions ionmolécule, peuvent être prédites par la théorie avec une précision largement suffisante.

Au début des années 90 , au contraire des réactions ion-molécule, les données concernant les réaction neutre-neutre en dessous de $200 \mathrm{~K}$ étaient extrêmement rares [9]. Pour cette raison, la nouvelle installation CRESU récemment construite à Rennes comprend une chambre d'expérience dédiée à l'étude de ce type de réactions. Cette chambre a été spécialement conçue pour permettre l'utilisation de la puissante technique dite PLP-LIF (Pulsed Laser Photolysis - Laser Induced Fluorescence). Ainsi, depuis 1991 , de très nombreuses réactions impliquant les radicaux $\mathrm{CN}, \mathrm{CH}, \mathrm{OH}$ et les atomes d'aluminium avec différentes molécules staḅles ont pu être étudiées. Les résultats obtenus montrent que les réactions radical-molécule sont souvent extrêmement rapides à très basse température, ce qui lève un jour nouveau sur les modèles de chimie interstellaire.

\section{DESCRIPTION DE L'APPAREILLAGE}

L'installation CRESU à Rennes a été conçue pour permettre soit l'étude des réactions ion-molécule, soit celle des réactions neutre-neutre. Sur la figure 1, un schéma d'ensemble montre les deux chambres d'expérience. L'ensemble est pompé par des pompes roots étagées dont la vitesse totale de pompage atteint $22500 \mathrm{~m}^{3} / \mathrm{h}$ dans l'hélium. Dans chaque chambre de nombreuses tuyères peuvent être aisément fixées sur le réservoir grâce à une bride à montage rapide permettant ainsi de diversifier les conditions de température et de pression de l'écoulement. Dans le cas des réactions neutre-neutre il est aussi possible de travailler à la température ambiante en ajustant la pression dans la chambre à une valeur suffisamment élevée pour obtenir un écoulement largement subsonique dans la tuyère. Pour obtenir les températures les plus basses $(13 \mathrm{~K})$, il est possible de refroidir les réservoirs à l'azote liquide.

Dans la chambre dédiée aux réactions neutre-neutre, deux faisceaux lasers pulsés sont combinés par un miroir dichroïque et se propagent suivant l'axe de l'écoulement. Dans la version actuelle, un laser $\mathrm{Nd}$ :Yag quadruplé $(266 \mathrm{~nm})$ est utilisé pour produire le radical à étudier par photolyse d'un précurseur adéquat; un deuxième laser accordable de 


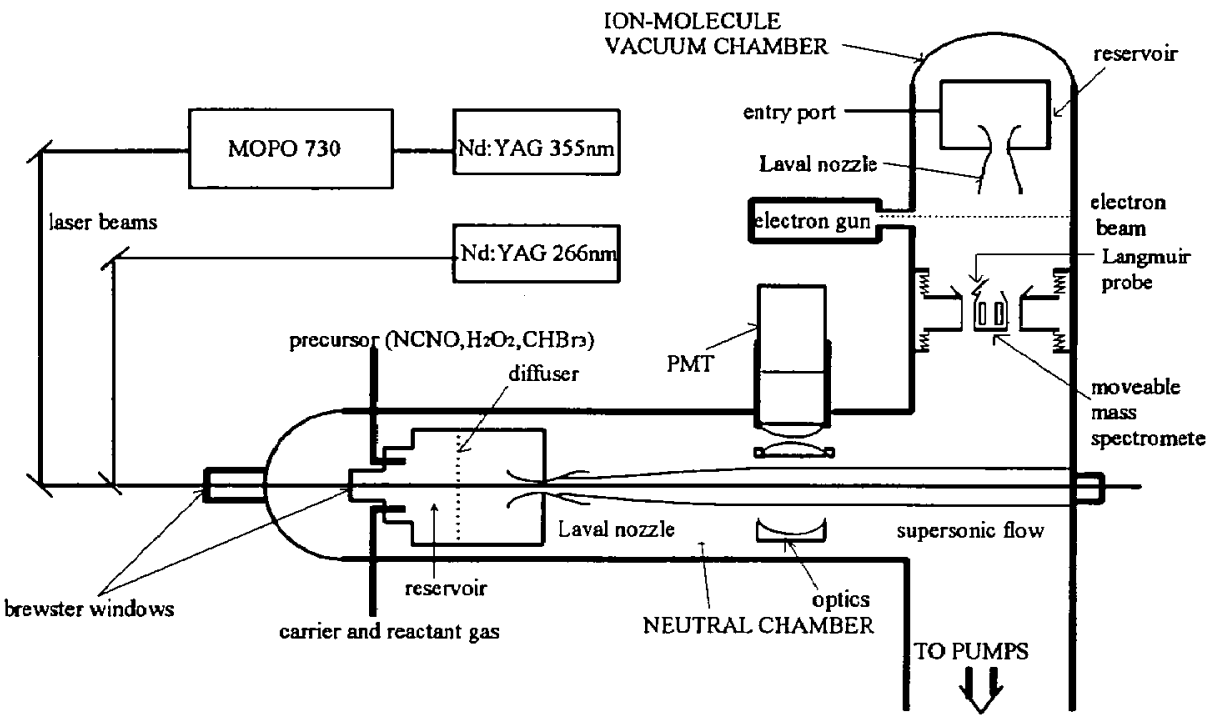

Figure 1: Schéma de l'appareillage CRESU de I'Université de Rennes I

$220 \mathrm{~nm}$ à $1.8 \mu \mathrm{m}$ (MOPO 730, Spectra Physics) permet la détection du radical par fluorescence induite. Le délai entre les deux impulsions lasers peut être ajusté entre 0 et quelques $\mathrm{ms}$ à quelques $\mathrm{ns}$ près. La lumière de fluorescence est collectée à une distance variable de la sortie de la tuyère $(4-45 \mathrm{~cm})$ en utilisant une optique à grande ouverture très efficace et un photomultiplicateur. La technique est donc très semblable aux expériences correspondantes en cellules, l'écoulement supersonique permettant seulement de fournir des conditions constantes de pression et de très basse température pendant un temps suffisamment long entre les deux impulsions lasers.

Quand un réactant est ajouté à l'écoulement, le signal de fluorescence décroît de façon exponentielle comme on peut le voir sur la figure 2 pour le cas du radical $\mathrm{OH}$ avec le trans-2-butène. Cette décroissance donne un coefficient de vitesse (en $\mathrm{s}^{-1}$ ) du pseudo premier ordre. En traçant la valeur de ce coefficient en fonction du débit de réactant (figure 3), on obtient une droite dont la pente donne le coefficient de vitesse bimoléculaire de la réaction étudiée.

Un problème très critique dans toute mesure à très basse température est celui des conditions thermodynamiques utilisées, en particulier en ce qui concerne la population des états internes. Le radical $\mathrm{CN}$ avec sa constante rotationnelle de $2.7 \mathrm{~K}$ est un excellent thermomètre et nous a permis de corroborer pour la température les valeurs déduites de mesures de pression d'impact (tube de Pitot) en supposant l'écoulement isentropique (figure 4). Dans ce cas, le radical était crée au seuil de photolyse et le spectre de rotation correspond très exactement à une distribution de Boltzmann même pour un délai entre les deux impulsions lasers extrêmement court. Les radicaux $\mathrm{OH}$ et $\mathrm{CH}$ sont par contre créés "chauds" en rotation et il est nécessaire d'attendre un temps suffisamment long pour une complète relaxation. Ceci est aussi vrai pour les états de spin-orbite comme nous l'avons observé très récemment pour le cas des atomes d'aluminium. 


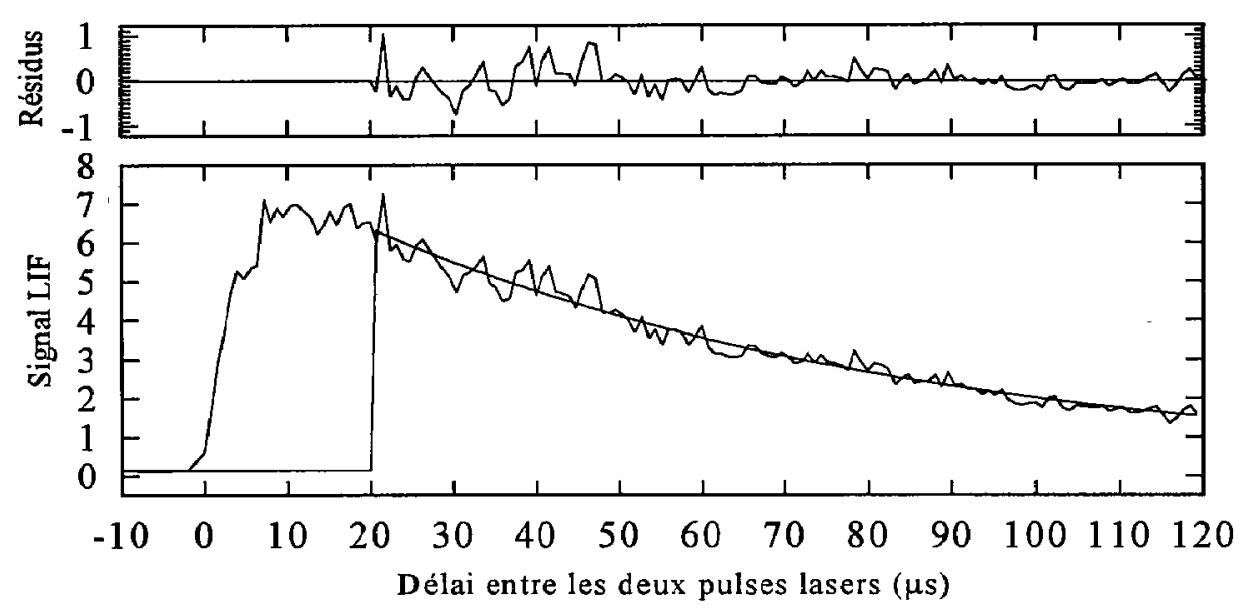

Figure 2: Décroissance du signal de fluorescence de $O H\left(X^{2} \Pi, v=0\right)$ en présence de trans-2-butène

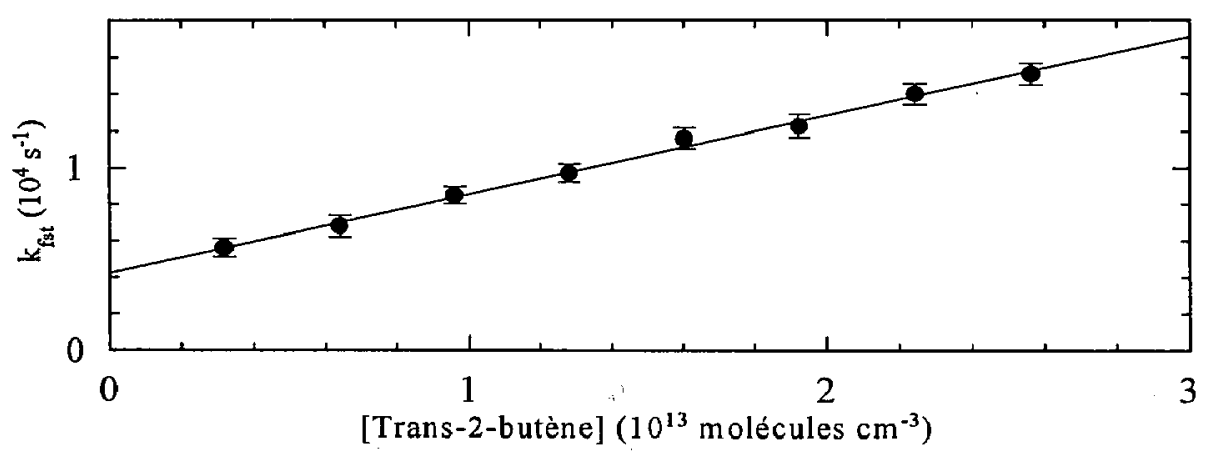

Figure 3: Tracé du coefficient de pseudo premier ordre en fonction de la densité de trans-2-butène. La pente de la droite donne le coefficient de vitesse recherché.

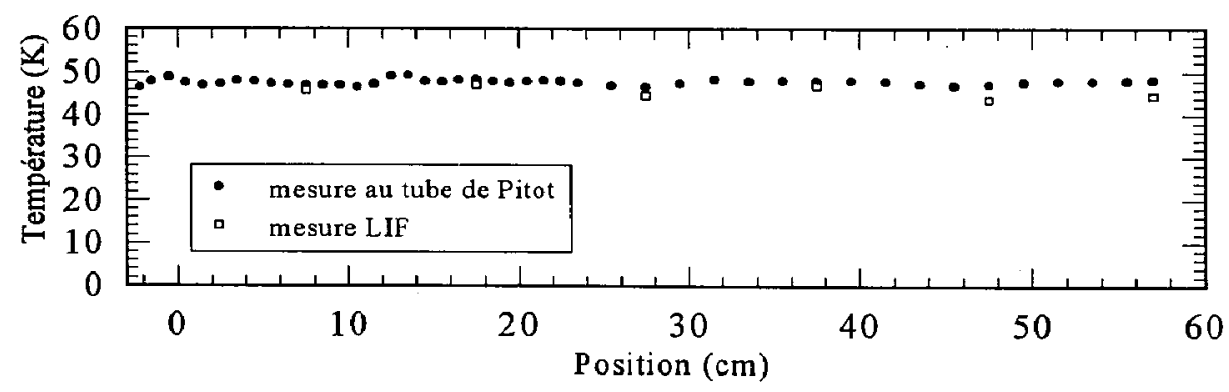

Figure 4: Profil de température de l'écoulement obtenu pour une tuyère fonctionnant dans l'azote. On notera l'excellent accord entre les mesures de type aérodynamique (Pitot) et les mesures par spectroscopie sur le radical $\mathrm{CN}$. 


\section{RESULTATS ET DISCUSSION}

La table 1 résume l'ensemble des processus radical-molécule étudiés à ce jour à Rennes. Il est bien entendu hors de question de détailler ici l'ensemble de ces résultats et nous renvoyons le lecteur intéressé aux nombreuses publications sur le sujet [10-17]. Toutes les réactions étudiées demeurent extrêmement rapides à très basse température ( 23 ou 13 $\mathrm{K})$ et ne présentent donc pas d'énergie d'activation.

Table 1: Ensemble des réactions radical-neutre étudiées grâce à l'installation CRESU de l'Université de Rennesl. Les valeurs numériques correspondent aux températures minimum auxquels ont été effectuées les mesures. Les réactions marquées d'une * sont des processus termoléculaires.

\begin{tabular}{|c|c|c|c|c|c|c|c|c|c|}
\hline Radical & \multicolumn{9}{|c|}{ Molécules Réactantes } \\
\hline $\begin{array}{l}\mathrm{CN} \\
\mathrm{T}_{\min }\end{array}$ & $\begin{array}{l}\mathrm{O}_{2} \\
13 \\
\end{array}$ & $\begin{array}{c}\mathrm{NH}_{3} \\
25\end{array}$ & $\begin{array}{c}\mathrm{C}_{2} \mathrm{H}_{2} \\
25\end{array}$ & $\begin{array}{c}\mathrm{C}_{2} \mathrm{H}_{4} \\
25\end{array}$ & $\begin{array}{c}\mathrm{C}_{2} \mathrm{H}_{6} \\
25\end{array}$ & & & & \\
\hline $\begin{array}{l}\mathrm{OH} \\
\mathrm{T}_{\min }\end{array}$ & $\begin{array}{c}\mathrm{HBr} \\
23\end{array}$ & \begin{tabular}{|c|} 
but-1-ène \\
$23^{*}$ \\
\end{tabular} & $\begin{array}{c}\text { Z-but-2-ène } \\
23^{*}\end{array}$ & $\begin{array}{c}\text { E-but-2-ène } \\
23^{*}\end{array}$ & $\begin{array}{l}\text { NO } \\
23^{*} \\
\end{array}$ & & & & \\
\hline $\begin{array}{l}\mathrm{CH}(\mathrm{v}=0) \\
\mathrm{T}_{\min }\end{array}$ & $\begin{array}{l}\mathrm{O}_{2} \\
13\end{array}$ & $\begin{array}{l}\text { NO } \\
13 \\
\end{array}$ & $\begin{array}{l}\mathrm{D}_{2} \\
13 \\
\end{array}$ & $\begin{array}{c}\mathrm{NH}_{3} \\
23\end{array}$ & $\begin{array}{c}\mathrm{CH}_{4} \\
23\end{array}$ & $\begin{array}{c}\mathrm{C}_{2} \mathrm{H}_{2} \\
23 \\
\end{array}$ & $\begin{array}{c}\mathrm{C}_{2} \mathrm{H}_{4} \\
23 \\
\end{array}$ & $\begin{array}{c}\mathrm{C}_{2} \mathrm{H}_{6} \\
23 \\
\end{array}$ & \begin{tabular}{|c|} 
but-1-ène \\
23 \\
\end{tabular} \\
\hline $\begin{array}{l}\mathrm{CH}(\mathrm{v}=0) \\
\mathrm{T}_{\min }\end{array}$ & $\begin{array}{c}\mathrm{H}_{2} \\
53^{*}\end{array}$ & $\begin{array}{l}\mathrm{CO} \\
53^{*} \\
\end{array}$ & $\begin{array}{r}\mathrm{N}_{2} \\
53^{*} \\
\end{array}$ & & & & & & \\
\hline $\begin{array}{l}\mathrm{CH}(\mathrm{v}=1) \\
\mathrm{T}_{\min }\end{array}$ & $\begin{array}{l}\mathrm{H}_{2} \\
23 \\
\end{array}$ & $\begin{array}{l}\mathrm{N}_{2} \\
23\end{array}$ & $\begin{array}{l}\mathrm{CO} \\
23 \\
\end{array}$ & $\begin{array}{l}D_{2} \\
23\end{array}$ & & & & & \\
\hline $\begin{array}{l}\mathrm{Al} \\
\mathrm{T}_{\min }\end{array}$ & $\begin{array}{l}\mathrm{O}_{2} \\
23\end{array}$ & & & & & & & & \\
\hline
\end{tabular}

En ce qui concerne les réactions bimoléculaires, trois types de comportement, illustrés par les figures 5,6 et 7 , ont été observé. Tout d'abord, le coefficient de vitesse peut augmenter continûment depuis la température ambiante jusqu'aux plus basses températures explorées. C'est le cas de la réaction $\mathrm{CN}+\mathrm{O}_{2}$ qui fut la toute première réaction étudiée avec cet appareillage. La figure 5 montre une augmentation d'un ordre de grandeur du coefficient de vitesse entre 760 et $13 \mathrm{~K}$. La variation du coefficient de vitesse est évidemment beaucoup moins prononcée lorsque sa valeur à $300 \mathrm{~K}$ est déjà proche de la limite collisionnelle (quelques $10^{-10} \mathrm{~cm}^{3} \mathrm{~s}^{-1}$ ) comme par exemple dans le cas des réactions de $\mathrm{CH}$ avec $\mathrm{NO}$ et $\mathrm{NH}_{3}$. Un autre type de comportement est caractérisé par l'augmentation du coefficient de vitesse suivie d'une décroissance lorsque la température décroît. Le coefficient de vitesse passe alors par un maximum correspondant à une température inférieure à $100 \mathrm{~K}$ (d'où le danger des extrapolations). C'est par exemple le cas des réactions de $\mathrm{CN}$ avec $\mathrm{C}_{2} \mathrm{H}_{4}$ ou de $\mathrm{CH}$ avec $\mathrm{C}_{2} \mathrm{H}_{4}$ (figure 6). Enfin, un cas très intéressant est celui de la réaction du radical $\mathrm{CN}$ avec l'éthane $\mathrm{C}_{2} \mathrm{H}_{6}$, molécule saturée (figure 7). Le coefficient de vitesse passe alors par un minimum puis croît pour atteindre une valeur proche de la limite collisionnelle à $23 \mathrm{~K}$. Ce résultat surprenant reste à expliquer sur le plan théorique.

La possibilité que les coefficients de vitesse mesurés pour les réactions bimoléculaires soient le fruit de l'association termoléculaire qui formerait des complexes de Van der Waals stabilisés peut être écartée. En effet, les mesures sont systématiquement effectuées en changeant la nature du gaz porteur (hélium, argon ou azote) et en utilisant différentes pressions pour vérifier la nature bimoléculaire des réactions. De plus, la formation de tels complexes serait beaucoup plus probable avec le gaz porteur (à l'exception de l'hélium) de par sa forte densité. La formation de complexes conduirait à une décroissance très rapide du signal de fluorescence, même en l'absence de gaz réactant, ce qui n'a jamais été observé. 


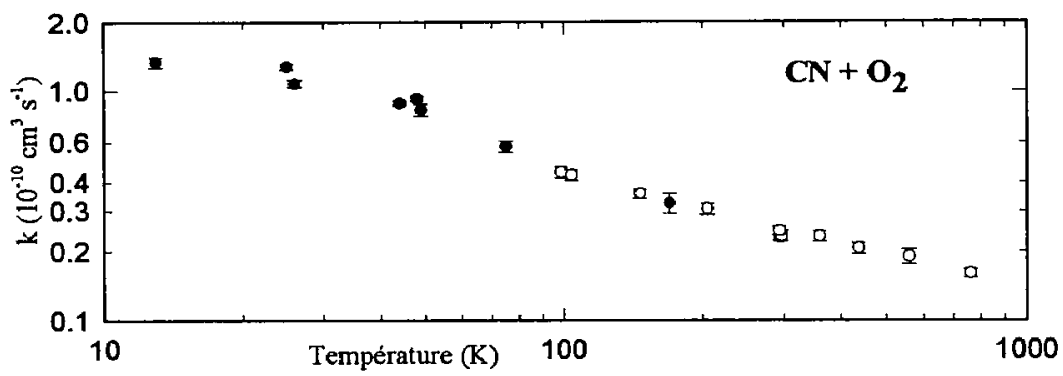

Figure 5: Evolution du coefficient de vitesse en fonction de la température pour $\mathrm{CN}+\mathrm{O}_{2}$ Les cercles noirs sont les mesures CRESU, les cercles blancs ont été obtenus dans une expérience en cellule (Sims and Smith, Chem. Phys. Lett., 151 (1988) 481).

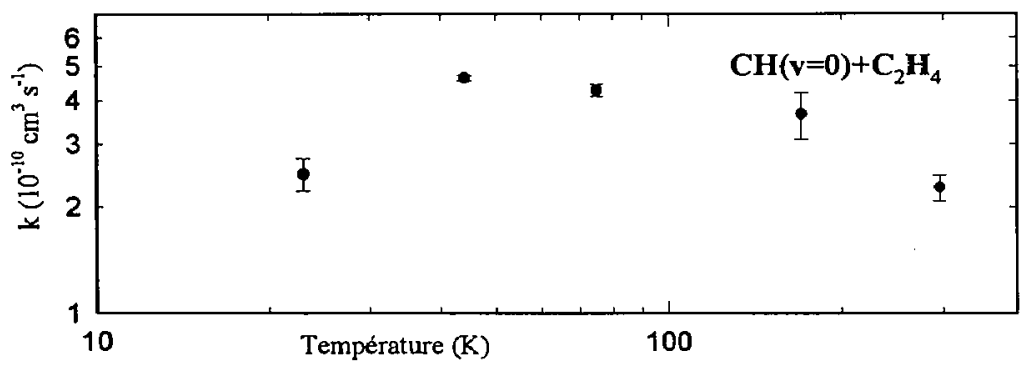

Figure 6: Evolution du coefficient de vitesse en fonction de la température pour $\mathrm{CH}+\mathrm{C}_{2} \mathrm{H}_{4}$

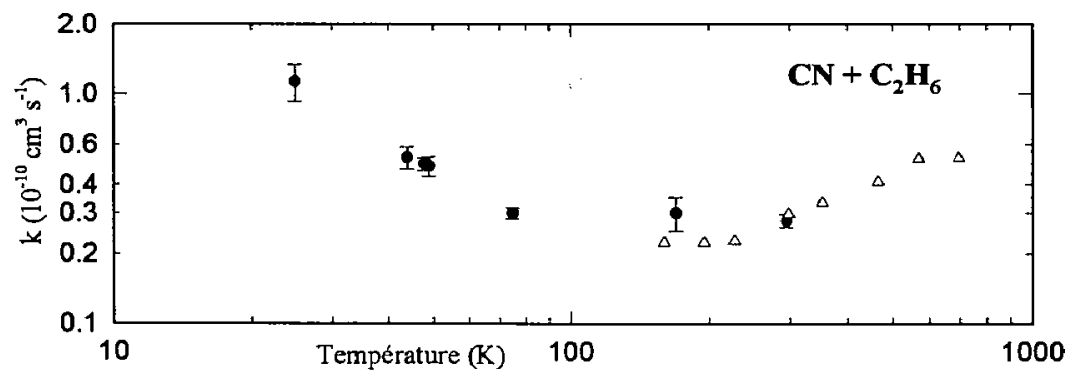

Figure 7: Evolution du coefficient de vitesse en fonction de la température pour $\mathrm{CN}+\mathrm{C}_{2} \mathrm{H}_{6}$ Les cercles noirs sont les mesures CRESU, les triangles blancs ont été obtenus dans une expérience en cellule [10].

Il est clair, au vu des résultats expérimentaux, qu'un très grand nombre de type de comportements cinétiques peuvent être observés à température ultra basse. Pour les réactions dont le coefficient de vitesse augmente ou présente un léger maximum quand la température décroît, on peut chercher à reproduire la variation du coefficient de vitesse en fonction de la température par des calculs théoriques de type capture qui n'utilisent que la partie à longue portée du potentiel intramoléculaire. Dans le cas des réactions ionmolécule ce type de calcul donne souvent un excellent accord avec l'expérience [18]. Le 
cas des réactions radical-neutre est bien plus complexe et des calculs effectués par $\mathbf{D}$. Clary et ses collaborateurs [19] antérieurement aux résultats expérimentaux en utilisant diverses variantes de la théorie de capture adiabatique ne donnent des résultats satisfaisants que pour le cas de $\mathrm{CH}+\mathrm{D}_{2}$. Plus récemment, $\mathrm{T}$. Stoecklin et ses collaborateurs [20] ont montré que les résultats sont extrêmement sensibles à la forme du potentiel intermoléculaire utilisé: un ajustement du terme lié aux forces de dispersion, dont le domaine de validité est probablement réduit à des distances intermoléculaires plus élevées que pour celui des contributions purement électrostatiques, permet de reproduire plus correctement les résultats expérimentaux. Il n'en demeure pas moins qu'un travail très important reste à effectuer pour obtenir des modèles collisionnels prédictifs à priori.

En ce qui concerne la nature des produits formés dans les réactions, problème bien sûr essentiel pour les applications, aucune étude systématique n'a encore été menée sur l'installation CRESU et pour le moment nous sommes donc amenés à les déduire d'étude effectuées à plus haute température. Ce type d'étude est beaucoup plus difficile à mener pour les réactions neutre-neutre que dans le cas des réactions ion-molécule où le spectromètre de masse renseigne directement sur les populations des ions présents dans l'écoulement. Cependant, il est probablement possible d'effectuer des études spécifiques pour les produits de réaction présentant des spectres de fluorescence adéquats.

Les réactions d'association termoléculaires présentent un intérêt évident pour certaines atmosphères planétaires telles que Titan ou Neptune par exemple. Ce type d'étude a pu être menée sur l'installation CRESU à $53 \mathrm{~K}$ pour des pressions variants de 0.4 mbar à 6 mbar pour plusieurs réactions (noté * dans la table 1). Complétées par des expériences en cellule effectuées à plus hautes températures par l'équipe du professeur I. Smith de l'Université de Birmingham, ces résultats ont permis de déterminer l'évolution en fonction de la température du coefficient de vitesse dans la limite de basse pression. Il a été d'autre part possible de conforter à partir de ces études des valeurs calculées du coefficient de vitesse pour l'association radiative processus supposé jouer un rôle important dans la chimie interstellaire. Nous avons par ailleurs également étudié la désexcitation vibrationnelle du radical $\mathrm{CH}$, processus qui permet de nous renseigner sur les mécanismes d'association radiative et collisionnelle de ce radical. En effet, la désexcitation vibrationnelle très efficace observée aux très basses températures peut s'expliquer par la formation d'un complexe transitoire fortement lié ${ }^{16}$, étape par laquelle passe aussi les deux mécanismes d'association précédents. Le complexe peut alors soit être stabilisé par émission de photon ou par un troisième corps (le gaz porteur), soit se redissocier par décomposition unimoléculaire. Dans le cas de la désexcitation vibrationnelle, cette dissociation conduit à un radical désexcité d'où la très grande efficacité du processus.

\section{CONCLUSIONS}

Pendant plus de 20 ans, les modèles de chimie interstellaire ont été essentiellement dépendants de la chimie ionique en phase gazeuse. Même si ces modèles incluaient quelques réactions neutre-neutre, les résultats obtenus à Rennes ont montré que cette chimie avaient été largement sous-estimée. De fait, les radicaux et les molécules que nous avons étudiés sont des espèces qui soit ont été détectées dans le MIS, soit y sont supposées abondantes. Cependant, il est clair que de très nombreuses réactions devront encore être étudiée expérimentalement avant de fonder la chimie interstellaire des espèces neutres sur des bases fermes. 


\section{Remerciements}

Les auteurs remercient l'ensemble de leur collaborateurs et tout particulièrement le professeur I. Smith et le docteur I. Sims de l'Université de Birmingham pour leur précieuse aide dans le développement et la mise en oeuvre des expériences. Nous remercions également le GDR "Physico-chimie des Molécules et des Grains Interstellaires" ainsi que le "Programme National de Planétologie" pour leur participation financière qui a favorisé la réalisation de ce travail.

\section{Références}

[1] Lewis J.S., "Cosmic Abundances Matter, AIP Conference Proceedings", 183, (1989) 17-37.

[2] Herbst E., Ann. Rev. Phys. Chem. 46 (1995) 27-53.

[3] Böhringer H and Arnold F., Int. J. Mass Spectrom. Ion. Proc. 49 (1983) 61.

[4] Barlow S.E., Luine J.A. and Dunn G.H., Int. J. Mass Spectrom. Ion. Proc. 74 (1986) 97.

[5] Gerlich D., State Selected and State to State Ion Molecule Reaction Dynamics, Part 1, C.Y. Ng and M. Baer Eds (John Wiley, New York, 1992) 1-176.

[6] Gerlich D., "XII th Symposium on Molecular Beams", Perugia, V. Aquilanti Ed., (1989) 37.

[7] Rowe B.R., Dupeyrat G., Marquette J.B. and Gaucherel P., J. Chem. Phys., 80 (1984) 4915.

[8] Hawley M., Mazely T.L., Randeniya, Smith R.S., Zeng X.K. and Smith M.A., Int. J. Mass Spectrom. Ion. Proc. 80 (1990) 239.

[9] National Institute of Standards and Technology, Chemical Kinetics Database 6.0

[10] Sims I.R., Queffellec J.L., Travers D., Rowe B.R., Herbert L., Karthauser J.and Smith I.W.M., Chem. Phys. Lett., 211 (1993) 461-468.

[11] Sims I.R., Queffellec J.L., Defrance A., Rebrion-Rowe C., Travers D., Bocherel P., Rowe B.R. and Smith I.W.M., J. Chem, Phys, 100 (1994) 4229-4241.

[12] Sims I.R., Bocherel P., Defrance A., Travers D., Rowe B.R. and Smith I.W.M., J. Chem. Soc. Faraday Trans. 90 (11) (1994) 1473-1478.

[13] Sims I.R., Smith I.W.M., Clary D.C., Bocherel P. and Rowe B.R., J. Chem. Phys., 101 (2) (1994) 1748-1751.

[14] Sharkey P., Sims I.R., Smith I.W.M, Bocherel P. and Rowe B.R., J. Chem. Soc. Faraday Trans., 90 (1994) 3609-3616.

[15] Bocherel P., Herbert L.B., Rowe B.R., Sims I.R., Smith I.W.M.and Travers D., J. Phys. Chem., 100 (1996) 3063-3069.

[16] Brownsword R.A., Herbert L., Sims I.R., Smith I.W.M., Stewart D.W.A., Symonds A.C., Canosa A. and Rowe B.R., J. Phys. Chem. accepté.

[17] Canosa A., Sims I.R., Travers D., Smith I.W.M. and Rowe B.R., Astronomy and Astrophysics (soumis).

[18] Rowe B.R. and Rebrion C., Trends in Chem. Phys., 1 (1991) 367-389.

[19] Clary D.C., J. Chem. Soc. Faraday Trans. 89 (1993) 2185.

[20] Stoecklin T. communication personnelle . 